\title{
BMJ Open Childhood academic ability in relation to cigarette, alcohol and cannabis use from adolescence into early adulthood: Longitudinal Study of Young People in England (LSYPE)
}

\author{
James Williams, ${ }^{1}$ Gareth Hagger-Johnson ${ }^{2}$
}

To cite: Williams J, HaggerJohnson G. Childhood academic ability in relation to cigarette, alcohol and cannabis use from adolescence into early adulthood: Longitudinal Study of Young People in England (LSYPE). BMJ Open 2017;7:e012989. doi:10.1136/bmjopen-2016012989

- Prepublication history and additional material is available. To view please visit the journal (http://dx.doi.org/ 10.1136/bmjopen-2016012989).

Received 9 June 2016 Revised 14 October 2016 Accepted 9 November 2016

CrossMark

${ }^{1}$ UCL Medical School, UCL, London, UK

${ }^{2}$ Administrative Data Research Centre England (ADRC-E), UCL, London, UK

Correspondence to Dr James Williams; james.williams.12@ucl.ac.uk

\section{ABSTRACT}

Objectives: Our aim was to determine the association between childhood academic ability and the onset and persistence of tobacco, alcohol and cannabis use across adolescence in a representative sample of English schools pupils. Previous research has produced conflicting findings.

Design: Data from 7 years of the Longitudinal Study of Young People in England (LSYPE), 2004-2010 (age 13/14-19/20).

Setting: Self-completion questionnaires during home visits, face-to-face interviews and web-based questionnaires.

Participants: Data from 6059 participants (3093 females) with information on academic ability around age 11 and health behaviours from age 13/14 to 16/17 (early adolescence) and from age 18/19 to 19/20 (late adolescence).

Outcome measures: Regularity of cigarette smoking, alcohol drinking and cannabis use from early to late adolescence.

Results: In multinomial logistic regression models adjusting for a range of covariates, the high (vs low) academic ability reduced the risk of persistent cigarette smoking ( $R R=0.62$; $\mathrm{Cl} 95 \% 0.48$ to 0.81 ) in early adolescence. High (vs low) academic ability increased the risk of occasional (RR=1.25; $\mathrm{Cl} 95 \% 1.04$ to 1.51 ) and persistent (RR=1.83; $\mathrm{Cl} 95 \% 1.50$ to 2.23 ) regular alcohol drinking in early adolescence and persistent (RR=2.28; $\mathrm{Cl} 95 \% 1.84$ to 2.82) but not occasional regular alcohol drinking in late adolescence. High academic ability was also positively associated with occasional (RR=1.83; $\mathrm{Cl} 95 \% 1.50$ to 2.23 ) and persistent ( $R R=1.83$; $\mathrm{Cl} 95 \% 1.50$ to 2.23 ) cannabis use in late adolescence.

Conclusions: In a sample of over 6000 young people in England, high childhood academic at age 11 is associated with a reduced risk of cigarette smoking but an increased risk of drinking alcohol regularly and cannabis use. These associations persist into early adulthood, providing evidence against the hypothesis that high academic ability is associated with temporary 'experimentation' with substance use.

\section{Strengths and limitations of this study}

- This study provided a large sample size from a cohort representative of an entire school year in England, followed seven times over 7 years.

- Data were only available on regularity of alcohol consumption rather than quantity, and no data on cigarette smoking were available after age 15/ 16.

- Results may not generalise to the $66 \%$ of independent school students without data on academic ability scores.

\section{INTRODUCTION}

Cigarette smoking, alcohol consumption and cannabis use are prevalent behaviours among adolescents. In 2004, $12 \%$ of 14-year olds in England were smoking cigarettes regularly, $23 \%$ were drinking alcohol at least weekly and $17 \%$ had tried cannabis. ${ }^{1}$ In 2014, these figures had fallen to $4 \%, 6 \%$ and $9 \%$, respectively. ${ }^{2}$ Despite the downward trend in use over the last decade, these risky health behaviours present a large problem in terms of public health as substance use is a risk factor for immediate and long-term health problems, ${ }^{3-10}$ as well as negative non-health outcomes such as poor educational and employment outcomes. ${ }^{11-18}$ The outcomes of cannabis use were found to be worsened by early onset and increased frequency of use. ${ }^{7} 19{ }^{20}$ Understanding the risk factors for adolescent substance use can inform public health policymaking and help target interventions for those in high-risk groups.

The role of academic ability in determining patterns of substance use is not clear. Academic ability has been shown to act as a valid proxy for cognitive ability or intelligence, where there is an emerging evidence 
base. ${ }^{21}$ The majority of studies evaluating the association between cognitive ability and cigarette smoking report that higher ability is associated with less smoking and higher rates of cessation among existing smokers. ${ }^{11} 17$ 22-26 The association between ability and alcohol is less clear. Two studies of data from a cohort study in Sweden showed that men with lower ability at age 18-21 had increased alcohol consumption (total intake and binge drinking). ${ }^{27}$ However, another study found no significant link between lower ability and increased alcohol use $\mathrm{e}^{22}$ and other studies have shown the opposite pattern, that higher ability age 11 is associated with higher alcohol consumption in adulthood. ${ }^{28} 29$ There is evidence that higher ability in childhood is associated with cannabis use, ${ }^{30}{ }^{31}$ but the extent to which this is explained by 'experimentation' (a temporary regular pattern of use followed by cessation) is not known, and some studies find higher ability is associated with less cannabis use. ${ }^{22}$

No study has evaluated academic ability at age 11 in relation to the onset and persistence of all three substances, from early to late adolescence and into early adulthood. The aim of our study was to determine the association between academic ability in childhood and the onset and persistence of regular cigarette smoking, alcohol drinking and cannabis use from early to late adolescence in a representative sample of English school pupils (the term regular referring to repeated use of a substance over a period of 12 months). This would answer for the first time whether ability was associated with 'experimentation' in early adolescence or if the association persists into later adolescence.

\section{METHODS}

\section{Data set}

The Longitudinal Study of Young People in England (LSYPE) is a prospective cohort study of English school pupils. ${ }^{32}$ The cohort was created to evaluate the transitions made by young people from secondary and tertiary education into adulthood. The cohort is a two-stage stratified random sample of schools and pupils, with oversampling of socioeconomically deprived schools (deprivation was measured by the proportion of students in receipt of free school meals-schools in the top quintile of this distribution were classified as deprived) and ethnic minority pupils. Eight hundred and thirty-eight maintained (non-fee-paying) schools were selected using the Pupil Level Annual Schools Census (PLASC) and then ordered within each deprivation stratum and thus stratified by region then admissions policy. Students were sampled from PLASC depending on ethnic group and school selection probabilities. Fifty-two independent schools (fee-paying) were selected using the School Level Annual Schools Census (SLASC) and stratified by percentage of pupils with five or more $A^{*}-\mathrm{C}$ GCSE grades within boarding status and gender. Students were sampled directly from school rolls. An average of 33.25 students were sampled from each school. Few schoollevel covariates were available and so were not included in the analysis. Survey weights are used to adjust results for the survey design. Pupils and parents were invited to participate by letter, using databases of schools to identify potential participants. At recruitment in 2004 (typical age 13/14, n=15 770), parents provided written informed consent. Participants provided their own consent from age $17 / 18$ to $19 / 20$ for repeated annual follow-ups until 2010. Sample sizes and response rates at each wave of the study are detailed in online supplementary appendix 1. Ethical approval for our study was not required because it involved secondary analysis of pseudonymised data from the UK Data Service. The first author had additional permission to use data on academic ability (usage number: 83740) from 2015 to 2017 as described below.

\section{General academic ability}

In England, pupils sit a National Curriculum Test around age 11 called 'Key Stage 2'. The Key Stage 2 score which summarises combined performance in English, Mathematics and Science (range 15, 36) has been shown to be a valid proxy for general cognitive ability at the same age $(r=0.83) .{ }^{21}$ We divided this score into tertiles in our analytic sample to create three academic ability groups: low (15.0-26.7), medium (26.829.9) and high (29.9-36.0).

\section{Cigarette smoking}

At age 13/14 (2004), 14/15 (2005) and 15/16 (2006), participants were asked the question "Do you ever smoke cigarettes at all?" ("Yes," "No") and if "Yes," to report the regularity of their smoking ("I have never smoked," "I have only ever tried smoking once," "I used to smoke sometimes but I never smoke a cigarette now," "I sometimes smoke cigarettes now but I don't smoke as many as one a week," "I usually smoke between one and six cigarettes a week," "I usually smoke more than six cigarettes a week"). We grouped participants into persistent, regular smokers (at least sometimes and every year observed), occasional regular smokers (at least sometimes for 1 or 2 years) and non-smokers (no regular smoking) from age $14 / 15$ to $15 / 16$.

\section{Regular alcohol drinking}

From age 13/14 (2004) to 16/17 (2007) and from age $18 / 19$ (2009) to $19 / 20$ (2010), participants were asked "Have you ever had a proper alcoholic drink?" ("Yes," "No"), and "Thinking about the last 12 months, about how often did you usually have an alcoholic drink?" ("Most days," "Once or twice a week," "2 or 3 times a month," "Once a month," "Once every couple of months," "Less often"). We grouped participants into regular alcohol drinkers (at least monthly) and nonregular drinkers (including non-drinkers). Participants were then classified as persistent regular alcohol drinkers (every year), occasional regular alcohol drinkers 
(one or more years but not every year) or never regular alcohol drinkers. This classification was performed separately for early adolescence (age 14/15-16/17) and late adolescence (age 18/19-19/20).

\section{Hazardous alcohol drinking}

Participants were asked, "On those days when you did have an alcoholic drink, how often would you say you got drunk?" followed by six response options (Every time, Most times, Around half the time, Less than half the time, Rarely, Never). This information was combined with alcohol drinking regularity to identify participants with hazardous levels of alcohol drinking (participants who reported drunkenness more than 52 times per year, broadly equivalent to drinking alcohol to intoxication more than once per week).

\section{Cannabis use}

From age 13/14 (2004) to $16 / 17$ (2007) and from age $18 / 19$ (2009) to $19 / 20$ (2010), participants were asked "Have you ever tried Cannabis even if only once?" ("Yes," "No"). At age 19/20, participants were also asked "Have you used cannabis in the last 12 months?" ("Yes," "No"). To calculate early and late cannabis use patterns, participants were grouped into cannabis users (reported use) versus non-cannabis users (no reported use) at age 14/ $15-16 / 17$ and age 18/19-19/20, respectively. Participants were then classified as early/late non-cannabis users (no use age 15-17/19-20), occasional cannabis users (use age $15-17 / 19-20$ but not consistently) or persistent cannabis users (use throughout age 15-17/19-20).

\section{Demographic covariates}

Age and sex were recorded at age 13/14. We tested for effect modification by sex, but results were not statistically significant $(p>0.05)$; therefore, males and females were analysed together, treating sex as a covariate. Parents of participants were asked the following question to assess for longstanding disability/illness/infirmity of the participant: "Does (text fill: name of sample young person) have any longstanding illness, disability or infirmity? By longstanding I mean anything that has troubled (text fill: name of sample young person) over a period of time or that is likely to affect him/her over a period of time?" ("Yes," "No"). An eight-category classification of the young person's self-reported ethnic group was used and from this, participants were classified as either 'White' (1) or 'Ethnic Minority' (0). Highest parental educational attainment was derived from seven categories and grouped into 'Degree level' (1) or 'Non-degree level' (0). Highest occupational social class was recorded on the eight-tier National Statistics Socioeconomic Classification (NS-SEC) and grouped into 'Professional' (1) or 'Non-professional' (0).

\section{Statistical analysis}

Descriptive analysis was first used to characterise the analytic sample and identify bivariate associations between academic ability and study variables using tests for linear trends across the three groups. Multinomial logistic regression models were used for the main analysis to determine the association between academic ability and two outcomes-occasional and persistent substance use (vs no use), separately for early and late adolescence. Relative risks were first minimally adjusted for age and sex, then additionally adjusted for longstanding disability/illness/infirmity, ethnic minority status, parental education and occupational social class. Sample weights were used to obtain correct SEs, allowing for the survey design at recruitment. In sensitivity analyses, we excluded participants already using any of the three substances at recruitment (age 13/14), in order to determine if existing patterns of use influenced results. We also reran the models using a larger analytic sample including those lost to follow-up in later adolescence, to determine if attrition biased the results. We also checked whether patterns differed for hazardous alcohol consumption at age 18/19-19/20, combining data on alcohol regularity and intoxication. Analysis was also repeated using quintiles of academic ability, and while the individual associations were strengthened, the trends remained the same, adding little to the analysis and interpretation of the results. Finally, we removed a small proportion of participants whose onset of substance use began in late adolescence, to evaluate whether results differed after excluding this subgroup. All analyses were performed in Stata V.12.1.

\section{RESULTS}

The analytic sample comprised 6059 participants with available data on academic ability, substance use from age $13 / 14$ to $19 / 20$ and covariates (figure 1 ). The excluded 9711 participants had a mean KS2 score of 26.09 , which falls into the lowest ability tertile. In comparison, the analytic sample's mean KS2 score of 27.86 (middle ability tertile), suggesting a small bias towards higher ability pupils being included in the analytic

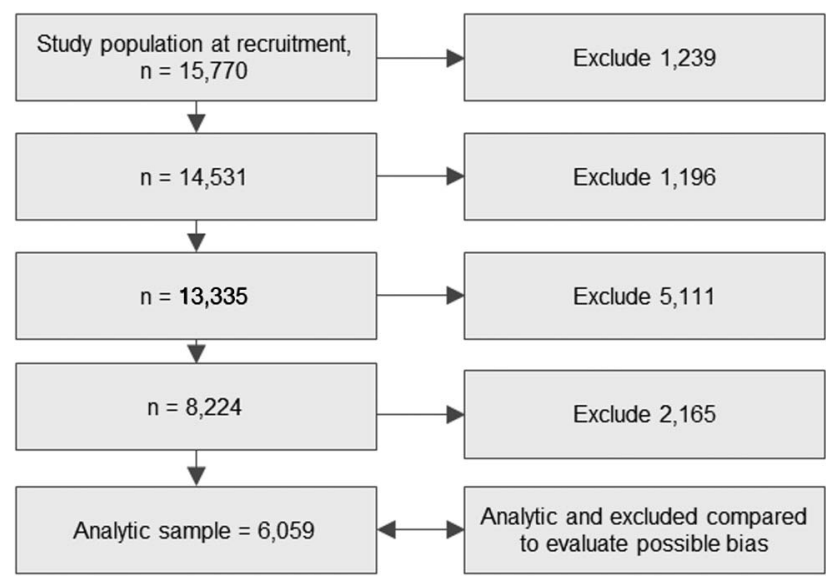

Figure 1 Flow diagram showing how the analytic sample was determined. 
sample. Compared with the study population at recruitment, the analytic sample comprised more females $(51.1 \%$ vs $48.1 \%)$, contained a smaller proportion of participants from a minority ethnic group $(28.5 \%$ vs $33.0 \%$ ) and a larger proportion of participants with a parent with degree level education $(18.0 \%$ vs $15.1 \%)$ or professional occupation $(45.7 \%$ vs $39.4 \%)$. Participants in the analytic sample from independent schools were slightly older, comprised fewer females $(46.5 \%)$ and contained a significantly smaller proportion of participants from an ethnic minority group (6.9\%) and a significantly larger proportion of participants with a parent with degree level education $(39.6 \%)$ or professional occupation (76.2\%). Participants from independent school comprised $3.4 \%(530 / 15770)$ of the total study population. Only $34 \%$ of these pupils had available KS2 data who comprised $1.7 \%$ of the analytic sample (101/ $6059)$, meaning that results may not generalise to feepaying schools.

Table 1 provides descriptive statistics of the analytic sample across three academic ability groups. The highest ability group was slightly older, with the highest proportion of males and White ethnicity and the highest proportion of participants without a longstanding disability/illness/infirmity and parental degree-level education or professional occupation. The highest ability group had the lowest proportion of early adolescent cigarette smokers but the highest proportion of regular alcohol drinkers and a similar proportion of cannabis smokers to the highest proportion medium ability group. There was a greater proportion of participants from independent schools in the high-ability group $(61.4 \%)$ with a smaller proportion persistently smoking cigarettes in early adolescence $(5.0 \%$ vs $8.2 \%)$ and a greater proportion persistently regularly drinking alcohol $(35.6 \%$ vs $26.0 \%)$ or using cannabis $(13.9 \%$ vs $13.0 \%)$ in early adolescence.

Online supplementary appendix 2 provides descriptive statistics of the analytic sample according to late adolescent substance use. Compared with the sample population, participants regularly drinking alcohol or using cannabis in late adolescence had a higher average academic ability score, were more likely to be male, White and have parents with a degree-level education or professional occupation.

\section{Early substance use (age 13-17)}

As shown in table 2, the high academic ability group was less likely to smoke cigarettes in early adolescence than the low-ability group, after adjustment for a range of covariates. This association was not significant for occasional smoking at one of the two study observations (adjusted $\mathrm{RR}=0.96$; $95 \%$ CI 0.74 to 1.23 ) but was significant for those smoking persistently at both observations (adjusted RR=0.62; 95\% CI 0.48 to 0.81 ). The association was weaker and non-significant for the medium (vs

Table 1 Descriptive statistics for study variables according to academic ability (unweighted)

\begin{tabular}{|c|c|c|c|c|c|}
\hline \multirow[b]{2}{*}{ Study variables } & \multicolumn{3}{|c|}{ Academic ability at age $11 / 12$} & \multirow[b]{2}{*}{ p Value } & \multirow[b]{2}{*}{$\begin{array}{l}\text { Total } \\
(\mathrm{N}=6059)\end{array}$} \\
\hline & $\begin{array}{l}\text { Low } \\
(15.00-26.74) \\
(\mathrm{N}=2024, \\
33.40 \%)\end{array}$ & $\begin{array}{l}\text { Medium } \\
(26.75-29.89) \\
(\mathrm{N}=2023 \text {, } \\
33.39 \%)\end{array}$ & $\begin{array}{l}\text { High } \\
(29.90-36.00) \\
(\mathrm{N}=2012, \\
33.21 \%)\end{array}$ & & \\
\hline$\overline{\text { Age, } M(S D)}$ & $14.21(0.30)$ & $14.23(0.31)$ & $14.28(0.31)$ & $<0.001$ & $14.24(0.31)$ \\
\hline Female, N (\%) & $1030(50.89)$ & $1077(53.24)$ & $986(49.01)$ & 0.027 & $3093(51.05)$ \\
\hline Longstanding illness, disability or infirmity, N (\%) & 299 (14.77) & $243(12.01)$ & 221 (10.98) & 0.001 & $763(12.59)$ \\
\hline Ethnic minority ${ }^{*}, \mathrm{~N}(\%)$ & $748(36.96)$ & 539 (26.64) & $438(21.77)$ & $<0.001$ & $1725(28.47)$ \\
\hline Parental occupation (non-professional), N (\%) & $1481(73.17)$ & 1070 (52.89) & 737 (36.63) & $<0.001$ & $3288(54.27)$ \\
\hline Parental education (non-degree level), N (\%) & 1875 (92.64) & 1709 (84.48) & $1382(68.69)$ & $<0.001$ & $4966(81.96)$ \\
\hline $\begin{array}{l}\text { Occasional early cigarette smoking age } \\
14-16, \mathrm{~N}(\%)\end{array}$ & $157(7.76)$ & $168(8.30)$ & $162(8.05)$ & 0.096 & $487(8.04)$ \\
\hline $\begin{array}{l}\text { Persistent early cigarette smoking age } \\
14-16, N(\%)\end{array}$ & $179(8.84)$ & $181(8.95)$ & $138(6.86)$ & 0.096 & $498(8.22)$ \\
\hline $\begin{array}{l}\text { Occasional early regular alcohol drinking age } \\
14-17, \mathrm{~N}(\%)\end{array}$ & $702(34.68)$ & 805 (39.79) & $801(39.81)$ & $<0.001$ & $2308(38.09)$ \\
\hline $\begin{array}{l}\text { Persistent early regular alcohol drinking age } \\
14-17, N(\%)\end{array}$ & $375(18.53)$ & $559(27.63)$ & $640(31.81)$ & $<0.001$ & $1574(25.98)$ \\
\hline Occasional early cannabis use age $14-17, \mathrm{~N}(\%)$ & $361(17.84)$ & $422(20.86)$ & $401(19.93)$ & $<0.001$ & $1184(19.54)$ \\
\hline Persistent early cannabis use age $14-17, \mathrm{~N}(\%)$ & $197(9.73)$ & 307 (15.18) & $283(14.07)$ & $<0.001$ & 787 (12.99) \\
\hline $\begin{array}{l}\text { Occasional later regular alcohol drinking age } \\
18-20, N(\%)\end{array}$ & $311(15.37)$ & $211(10.43)$ & $157(7.80)$ & $<0.001$ & $679(11.21)$ \\
\hline $\begin{array}{l}\text { Persistent later regular alcohol drinking age } \\
18-20, N(\%)\end{array}$ & $1061(52.42)$ & $1403(69.35)$ & $1582(78.63)$ & $<0.001$ & $4046(66.78)$ \\
\hline Occasional later cannabis use age $18-20, \mathrm{~N}(\%)$ & $298(14.72)$ & $393(19.43)$ & $435(21.62)$ & $<0.001$ & $1126(18.58)$ \\
\hline Persistent later cannabis use age $18-20, \mathrm{~N}(\%)$ & $215(10.62)$ & $351(17.35)$ & $407(20.23)$ & $<0.001$ & $973(16.06)$ \\
\hline
\end{tabular}




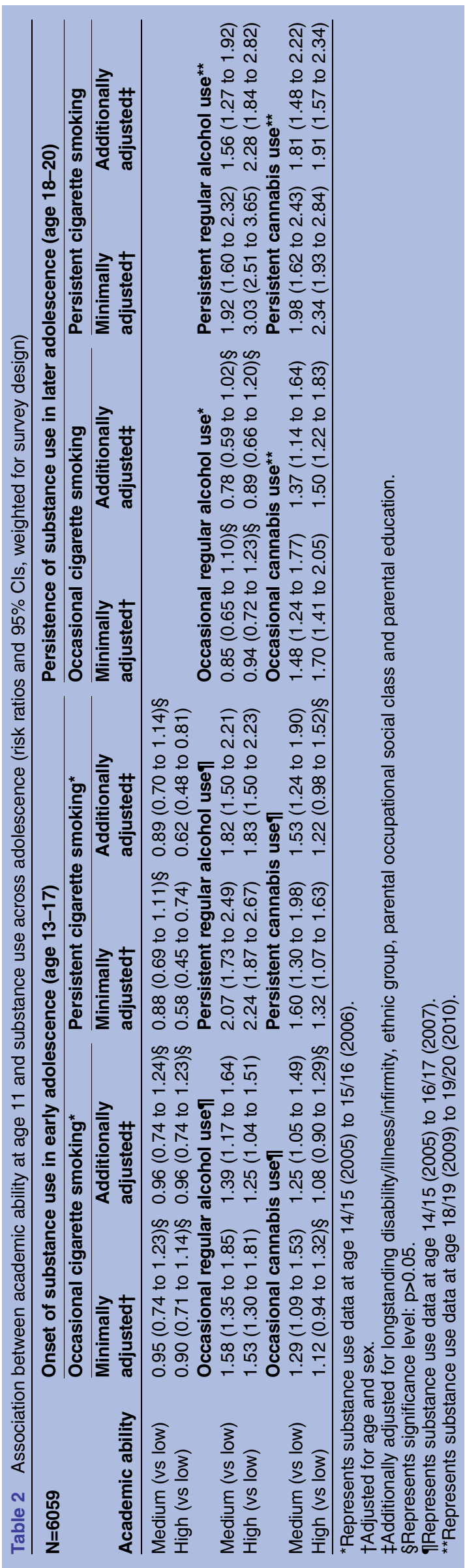

low) ability group, for occasional (adjusted $\mathrm{RR}=0.96$, 95\% CI 0.74 to 1.24 ) and persistent smoking (adjusted $\mathrm{RR}=0.89,95 \%$ CI 0.70 to 1.14 ).

The high-ability group was more likely to drink alcohol regularly in early adolescence than the lowability group. This was seen for occasional (adjusted $\mathrm{RR}=1.25$; $95 \%$ CI 1.04 to 1.51 ) and persistent (adjusted $\mathrm{RR}=1.83 ; 95 \%$ CI 1.50 to 2.23 ) alcohol drinking.

An association between the high (vs low) ability group and a greater likelihood of using cannabis in early adolescence was also seen, but this was not significant for occasional (adjusted $\mathrm{RR}=1.08,95 \%$ CI 0.90 to 1.29 ) or persistent (adjusted $\mathrm{RR}=1.22,95 \%$ CI 0.98 to 1.52 ) use. However, the medium-ability group were found to be more likely to use cannabis than the low-ability group, occasionally (adjusted $\mathrm{RR}=1.25$; 95\% CI 1.05 to 1.49 ) and persistently (adjusted $\mathrm{RR}=1.53 ; 95 \%$ CI 1.24 to 1.90). This suggests a potential non-linear association between academic ability and cannabis use in early adolescence, but as the CIs overlap, caution must be taken when interpreting this result.

\section{Late substance use (age 18-20)}

The associations between the high (vs low) academic ability and regular alcohol consumption and cannabis use seen in early adolescence persisted into late adolescence. Participants in the high (vs low) ability group were more than twice as likely to drink alcohol regularly and persistently (adjusted RR=2.28; 95\% CI 1.84 to 2.82). Patterns were weaker but very similar for the medium (vs low) ability group (adjusted RR $=1.56$; 95\% CI 1.27 to 1.92), suggesting a graded association between levels of ability and regular alcohol consumption. There was no significant association between high (adjusted $\mathrm{RR}=0.89$, $95 \%$ CI 0.66 to 1.20 ) or medium (adjusted $\mathrm{RR}=0.78,95 \%$ CI 0.59 to 1.02 ) ability and occasional alcohol use, compared with the low-ability group.

For late adolescent cannabis use, a similar graded association was seen between the high and medium (vs low) ability groups. Participants in the high (vs low) ability group were more likely to use cannabis occasionally (adjusted RR=1.50; 95\% CI 1.22 to 1.83 ) and persistently (adjusted RR=1.91; 95\% CI 1.57 to 2.34) in late adolescence, as were those in the medium (vs low) ability group (adjusted RR for occasional use $=1.37$; 95\% CI 1.14 to 1.64; adjusted RR for persistent use $=1.81$; $95 \%$ CI 1.48 to 2.22 ).

In sensitivity analyses, patterns were the same and associations were stronger after excluding participants $(n=1414)$ already using at least one of the three substances at recruitment (see online supplementary appendix 3). This suggests that the associations do apply to the onset of substance use in adolescence, and are not influenced by existing users. Results were not affected when rerunning models including 2165 participants who were lost to follow-up from age 18 to 20 , although associations were slightly stronger (see online supplementary appendix 4). This suggests that attrition 
may have led us to underestimate slightly the effect sizes we observed within the analytic sample. Results were not materially different after excluding participants with onset of substance use in late adolescence $(n=683)$ (see online supplementary appendix 5). High ability was associated with being less likely to engage in hazardous alcohol consumption, suggesting that to the regularity of consumption has the opposite association with ability than hazardous consumption (see online supplementary appendix 6). We also repeated the analyses with higher cut-off points for determining regularity of substance use, and found that the associations were slightly stronger, but with little change to overall patterns of results or conclusions drawn.

\section{DISCUSSION}

In a representative sample of more than 6000 young people in England, children with high academic ability at age 11 were less likely to persistently smoke cigarettes in early adolescence (from age 13 to 17 ) compared with those in the low-ability group. In contrast, they were more than twice as likely to drink alcohol regularly, occasionally or persistently across three study observations. Those with medium or high ability were more likely to use cannabis occasionally or persistently, but the association was not significant for the high-ability group. Medium or high ability at age 11 were both associated with regular and persistent alcohol use in later adolescence (from age 18 to 20 ), and with occasional and persistent cannabis use.

Strengths of the study include the large sample size, representative of an entire school year at recruitment, with seven follow-ups from age 13 to 20 . This allowed us to consider the association between academic ability at age 11 and the onset and persistence of substance use across adolescence. Results may not generalise to independent schools, where only $34 \%$ had available data on Key Stage 2 scores, or to schools in other countries.

One limitation of the study is the lack of detail available on substance use. For instance, regularity of cannabis use was not available until age 18-20. No data on quantity of alcohol typically consumed, nor amount of cannabis use were available. Use may be regular but light or sporadic but heavy and/or hazardous. This is potentially important, given the known association between lower cognitive ability and more hazardous or 'binge drinking' seen in adults. ${ }^{28} \mathrm{~A}$ further limitation is that data on cigarette smoking were not available after age 16. There is potential for biased estimates, given that the analytic sample was significantly older and comprised more pupils with high socioeconomic status. We did evaluate these differences however, and did consider loss to follow-up for the later adolescent period. Low ability pupils will be under-represented in our analytic sample (and in those recruited to the study population) which will have led us to underestimate the associations observed. Finally, the association between childhood academic ability and comorbid use of all three substances was not analysed in this study. Comorbid use of cigarettes, alcohol and cannabis is a common occurrence in the adolescent population ${ }^{33}$ and a recent analysis of a New Zealand cross-sectional study found that low academic ability was associated with a greater incidence of comorbid substance use in 14-15-year olds. ${ }^{34}$ The fact that we observed quite different associations between academic ability and smoking, alcohol and cannabis provides support for our decision to consider them individually.

Our finding that adolescents with high academic ability are less likely to smoke but more likely to drink alcohol regularly and use cannabis is broadly consistent with the evidence base on adults. ${ }^{11} 17$ 22-26 $28-31$ The reason for these associations is not fully understood, but several possible explanations have been proposed. Although cognitive ability is broadly associated with better life chances ${ }^{23}{ }^{24}$ and healthier life choices, ${ }^{31} 35$ cognitive ability is positively correlated with the personality trait 'openness to experience' which might encourage high-ability children to experiment with alcohol or cannabis. ${ }^{28} 3136$ The fact that this association persisted across the entire adolescent period and was strongest for persistent use, however, provides evidence against this hypothesis. Another explanation is that more able children are known to be accepted by older peers, who might have access to cannabis. ${ }^{31}$ Higher ability children might also be more honest in their self-reported behaviour, ${ }^{37}$ which we could not evaluate in this study. It is also possible that low-ability children lacked the literacy skills to recognise 'cannabis' as a drug they had used, although 14 commonly used synonyms were provided (weed, hash etc). Parental influence might partly explain the association with alcohol, since parents with high cognitive ability and socioeconomic status are known to drink alcohol more regularly (although less hazardously). ${ }^{38}$ Adults with high ability are more likely to respond to public health advice not to smoke, which they may share with their children. The finding that the association between academic ability and cannabis use in early adolescence was non-linear is unusual. A potential explanation for this is that perhaps high-ability adolescents are more likely to experiment but are initially cautious of illegal substances in early adolescence as they are more aware of the immediate and long-term repercussions that breaking the law may incur than those with lower academic ability. Further to this point, available data on KS2 results are limited. For each student, a score was given for each individual subjectMaths, English and Science-as well as an average, but subscales of KS2 results (eg, verbal ability in English) were not available within the first author's data access agreement from UK Data Service. This is a limitation as subscales of individual subjects may play different roles in the associations observed in the results. Subject-specific associations should be considered in future research. 
A major limitation of this paper is the observational design of the study, which has a limited range of possible confounding factors that could be considered. As outlined above, there are many factors that may play a role in the complex relationship between childhood academic ability and adolescent substance use, and it is not possible to explain the underlying mechanisms responsible for the results seen here using this cohort. Additionally, unlike previous cohort studies, ${ }^{39-41}$ the cohort cannot be used to separate age, period and cohort effects, because the pupils all share the same academic birth year. The second cohort (LSYPE2) will soon provide an opportunity to draw comparisons between pupils of the same age, in a later period.

Our results are relevant to clinicians and policymakers who are concerned about the impact of substance use on health and on educational outcomes. A greater understanding of substance use patterns in adolescence can help clinicians formulate appropriate treatment plans for young people and establish treatment goals to minimise use of substances and their negative sequelae. ${ }^{42}$ Although children with high academic ability are more likely to achieve good examination grades and entry into high status occupations, substance use could reduce their performance relative to their peers. ${ }^{10-17}$ It should not be assumed that children with low academic ability are at higher risk for substance use, despite the fact that this group of pupils are more likely to develop adverse health outcomes and misuse various substances, and engage in hazardous (rather than regular) alcohol consumption as seen here and elsewhere. ${ }^{27} 43$ Reducing harmful substance use in this age group is important, no matter the level of academic ability, given the immediate risks to health and the longer term consequences. For example, underage drinking of alcohol is associated with numerous adverse consequences, such as vomiting and coma, liver disease, involvement in road traffic accidents, depression, brain damage, dependency, educational performance and risky sexual practices. ${ }^{44}$ Despite reductions in the proportion of young people reporting substance use in recent years, these are still prevalent health behaviours. ${ }^{1}{ }^{2}$ As illustrated in this study, the association between high academic ability in childhood and substance use persists in late adolescence/early adulthood, posing a real challenge for clinicians and policymakers, with $22 \%$ of males and $16 \%$ of females aged 16 and over engaging in risky alcohol drinking. ${ }^{45}$ Further research should be conducted to further understand the relationship between childhood academic ability and adolescent substance use, which might offer insights into ways to prevent harm for all young people as they transition into adulthood. These studies should look at the quantity and context of tobacco, alcohol and cannabis use, as well as looking at the types of alcohol consumed and patterns of cessation throughout adolescence. Participants from LSYPE have been recruited for follow-up at the age of 25 and data are currently being collected for publication in 2017. ${ }^{46}$ This follow-up data, and the LSYPE2 cohort, will offer further opportunities to evaluate the relationship between ability and substance use and how it tracks into adulthood.

Contributors JW conducted the analysis. Both authors contributed to writing of the manuscript.

Funding This research received no specific grant from any funding agency in the public, commercial or not-for-profit sectors.

Competing interests None declared.

Ethics approval The study involved analysis of existing data at the UK Data Archive.

Provenance and peer review Not commissioned; externally peer reviewed.

Data sharing statement Data are available from the UK Data Archive. Syntax to reproduce the results presented here is available from the corresponding author on request.

Open Access This is an Open Access article distributed in accordance with the Creative Commons Attribution Non Commercial (CC BY-NC 4.0) license, which permits others to distribute, remix, adapt, build upon this work noncommercially, and license their derivative works on different terms, provided the original work is properly cited and the use is non-commercial. See: http:// creativecommons.org/licenses/by-nc/4.0/

\section{REFERENCES}

1. Health \& Social Care Information Centre. Smoking, drinking and drug use among young people in England in 2004. Leeds: Health and Social Care Information Centre, 2005.

2. Health \& Social Care Information Centre. Smoking, drinking and drug use among young people in England in 2014. Leeds: Health and Social Care Information Centre, 2015.

3. Peto R, Lopez AD, Boreham J, et al. Mortality from tobacco in developed countries: indirect estimation from national vital statistics. Lancet 1992;339:1268-78.

4. Warren KR, Murray MM. Alcoholic liver disease and pancreatitis: global health problems being addressed by the US National Institute on Alcohol Abuse and Alcoholism. J Gastroenterol Hepatol 2013;28 (Suppl 1):4-6.

5. Isenberg-Grzeda E, Kutner HE, Nicolson SE. Wernicke-Korsakoffsyndrome: under-recognized and under-treated. Psychosomatics 2012;53:507-16.

6. Chadwick B, Miller ML, Hurd YL. Cannabis use during adolescent development: susceptibility to psychiatric illness. Front Psychiatry 2013;4:129.

7. Hall W. The adverse health effects of cannabis use: what are they, and what are their implications for policy? Int J Drug Policy 2009;20:458-66.

8. Swift W, Coffey C, Carlin JB, et al. Adolescent cannabis users at 24 years: trajectories to regular weekly use and dependence in young adulthood. Addiction 2008;103:1361-70.

9. DeLisi LE. The effect of cannabis on the brain: can it cause brain anomalies that lead to increased risk for schizophrenia? Curr Opin Psychiatry 2008;21:140-50.

10. Parakh P, Basu D. Cannabis and psychosis: have we found the missing links? Asian J Psychiatr 2013;6:281-7.

11. Latvala A, Rose RJ, Pulkkinen L, et al. Drinking, smoking, and educational achievement: cross-lagged associations from adolescence to adulthood. Drug Alcohol Depend 2014;137:106-13.

12. Silins E, Fergusson DM, Patton GC, et al. Adolescent substance use and educational attainment: an integrative data analysis comparing cannabis and alcohol from three Australasian cohorts. Drug Alcohol Depend 2015;156:90-6.

13. Meier MH, Hill ML, Small PJ, et al. Associations of adolescent cannabis use with academic performance and mental health: a longitudinal study of upper middle class youth. Drug Alcohol Depend 2015;156:207-12.

14. Homel J, Thompson K, Leadbeater B. Trajectories of marijuana use in youth ages 15-25: implications for postsecondary education experiences. J Stud Alcohol Drugs 2014;75:674-83.

15. Kelly $A B$, Evans-Whipp TJ, Smith R, et al. A longitudinal study of the association of adolescent polydrug use, alcohol use and high school non-completion. Addiction 2015;110:627-35. 
16. Lynskey MT, Coffey C, Degenhardt L, et al. A longitudinal study of the effects of adolescent cannabis use on high school completion. Addiction 2003;98:685-92.

17. Fergusson DM, Horwood LJ, Ridder EM. Show me the child at seven II: childhood intelligence and later outcomes in adolescence and young adulthood. J Child Psychol Psychiatry 2005;46:850-8.

18. Horwood LJ, Fergusson DM, Hayatbakhsh MR, et al. Cannabis use and educational achievement: findings from three Australasian cohort studies. Drug Alcohol Depend 2010;110:247-53.

19. Fothergill KE, Ensminger ME, Green KM, et al. Pathways to adult marijuana and cocaine use: a prospective study of African Americans from age 6 to 42. J Health Soc Behav 2009;50:65-81.

20. Jackson $C$, Dickinson D. Cigarette consumption during childhood and persistence of smoking through adolescence. Arch Pediatr Adolesc Med 2004;158:1050-6.

21. Calvin CM, Deary IJ, Webbink D, et al. Multivariate genetic analyses of cognition and academic achievement from two population samples of 174,000 and 166,000 school children. Behav Genet 2012;42:699-710.

22. Harakeh Z, de Looze ME, Schrijvers CT, et al. Individual and environmental predictors of health risk behaviours among Dutch adolescents: the HBSC study. Public Health 2012;126:566-73.

23. Batty GD, Deary IJ, Schoon I, et al. Mental ability across childhood in relation to risk factors for premature mortality in adult life: the 1970 British Cohort Study. J Epidemiol Community Health 2007;61:997-1003.

24. Batty GD, Deary IJ, Macintyre S. Childhood IQ in relation to risk factors for premature mortality in middle-aged persons: the Aberdeen Children of the 1950s study. J Epidemiol Community Health 2007;61:241-7.

25. Solberg LI, Asche SE, Boyle R, et al. Smoking and cessation behaviors among young adults of various educational backgrounds. Am J Public Health 2007;97:1421-6.

26. Taylor MD, Hart CL, Davey Smith G, et al. Childhood mental ability and smoking cessation in adulthood: prospective observational study linking the Scottish Mental Survey 1932 and the Midspan studies. J Epidemiol Community Health 2003;57:464-5.

27. Sjölund S, Hemmingsson T, Allebeck P. IQ and level of alcohol consumption-findings from a national survey of Swedish conscripts. Alcohol Clin Exp Res 2015;39:548-55.

28. Batty GD, Deary IJ, Schoon I, et al. Childhood mental ability and adult alcohol intake and alcohol problems: the 1970 British cohort study. Am J Public Health 2008;98:2237-43.

29. Luczak SE, Yarnell LM, Prescott CA, et al. Childhood cognitive measures as predictors of alcohol use and problems by mid-adulthood in a non-Western cohort. Psychol Addict Behav 2015;29:365-70.

30. Storr CL, Wagner FA, Chen CY, et al. Childhood predictors of first chance to use and use of cannabis by young adulthood. Drug Alcohol Depend 2011;117:7-15.
31. White J, Batty GD. Intelligence across childhood in relation to illegal drug use in adulthood: 1970 British Cohort Study. J Epidemiol Community Health 2012;66:767-74.

32. Department for Education, NatCen Social Research. First longitudinal study of young people in England: waves one to seven, 2004-2010: secure access. [data collection]. 2nd edn. UK Data Service, 2012. SN: 7104.

33. Costello MJ, Leatherdale ST, Ahmed R, et al. Co-morbid substance use behaviors among youth: any impact of school environment? Glob Health Promot 2012;19:50-9.

34. White J, Walton D, Walker N. Exploring comorbid use of marijuana, tobacco, and alcohol among 14 to 15-year-olds: findings from a national survey on adolescent substance use. BMC Public Health 2015;15:233.

35. Batty GD, Deary IJ, Schoon I, et al. Childhood mental ability in relation to food intake and physical activity in adulthood: the 1970 British Cohort Study. Pediatrics 2007;119:e38-45.

36. Harris JA. Measured intelligence, achievement, openness to experience, and creativity. Pers Individ Dif 2004;36:913-29.

37. Austin EJ, Deary IJ, Whiteman MC, et al. Relationships between ability and personality: does intelligence contribute positively to personal and social adjustment? Pers Individ Dif 2002;32:1391-411.

38. Huckle T, You RQ, Casswell S. Socio-economic status predicts drinking patterns but not alcohol-related consequences independently. Addiction 2010;105:1192-202.

39. Deary IJ, Gow AJ, Pattie A, et al. Cohort profile: the Lothian birth cohorts of 1921 and 1936. Int J Epidemiol 2012;41:1576-84.

40. Wadsworth M, Kuh D, Richards M, et al. Cohort profile: the 1946 National Birth Cohort (MRC National Survey of Health and Development). Int J Epidemiol 2006;35:49-54.

41. Elliott J, Shepherd P. Cohort profile: 1970 British Birth Cohort (BCS70). Int J Epidemiol 2006;35:836-43.

42. Department of Health (England) and the devolved administrations. Drug misuse and dependence: UK guidelines on clinical management. London: Department of Health (England), the Scottish Government, Welsh Assembly Government and Northern Ireland Executive, 2007.

43. Sjölund $S$, Allebeck $P$, Hemmingsson T. Intelligence quotient (IQ) in adolescence and later risk of alcohol-related hospital admissions and deaths-37-year follow-up of Swedish conscripts. Addiction 2012;107:89-97.

44. Newbury-Birch D, Gilvarry E, McArdle P, et al. The impact of alcohol consumption on young people: a review of reviews. University of Newcastle, Institute of Health and Society, 2009.

45. Health \& Social Care Information Centre. Health survey for England, 2014. Leeds: Health and Social Care Information Centre, 2015.

46. Calderwood L, Sanchez C. Next steps (formerly known as the Longitudinal Study of Young People in England). Open Health Data $2016: 4: e 2$ 


\section{Correction}

Williams J, Hagger-Johnson G. Childhood academic ability in relation to cigarette, alcohol and cannabis use from adolescence into early adulthood: Longitudinal Study of Young People in England (LSYPE). BMJ Open 2017;7:e012989. doi: 10.1136/ bmjopen-2016-012989

There are some errors in the RR and 95\% CI values in the Results paragraph of the Abstract. This paragraph should read as follows:

\section{RESULTS}

In multinomial logistic regression models adjusting for a range of covariates, the high (vs low) academic ability reduced the risk of persistent cigarette smoking ( $\mathrm{RR}=0.62$; CI 95\% 0.48 to 0.81 ) in early adolescence. High (vs low) academic ability increased the risk of occasional ( $\mathrm{RR}=1.25$; CI 95\% 1.04 to 1.51 ) and persistent ( $\mathrm{RR}=1.83$; CI $95 \%$ 1.50 to 2.23) regular alcohol drinking in early adolescence and persistent ( $R R=2.28$; CI $95 \% 1.84$ to 2.82 ) but not occasional regular alcohol drinking in late adolescence. High (vs low) academic ability was also positively associated with occasional ( $R R=1.50$; CI $95 \% 1.22$ to 1.83 ) and persistent ( $\mathrm{RR}=1.91$; CI $95 \% 1.57$ to 2.34$)$ cannabis use in late adolescence.

Open Access This is an Open Access article distributed in accordance with the Creative Commons Attribution Non Commercial (CC BY-NC 4.0) license, which permits others to distribute, remix, adapt, build upon this work non-commercially, and license their derivative works on different terms, provided the original work is properly cited and the use is non-commercial. See: http://creativecommons.org/licenses/by-nc/4.0/

BMJ Open 2017;1:e012989corr1. doi:10.1136/bmjopen-2016-012989corr1

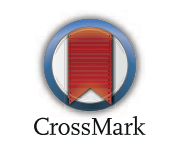

